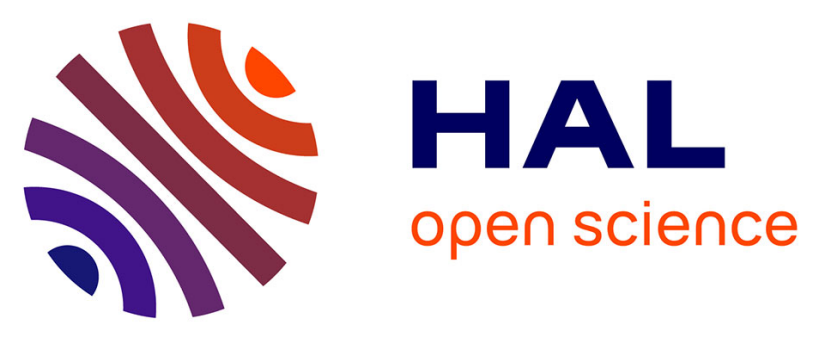

\title{
Exposition des chirurgiens et des patients aux rayonnements ionisants pendant les procédures d'ostéosynthèse rachidienne postérieure percutanée : étude prospective de 100 patients
}

Pascal Kouyoumdjian, Guillaume Gras-Combe, Mickael Grelat, Stephane Fuentes, Benjamin Blondel, Patrick Tropiano, Fahed Zairi, Jacques Beaurain, Yann-Philippe Charles, Alexandre Dhenin, et al.

\section{- To cite this version:}

Pascal Kouyoumdjian, Guillaume Gras-Combe, Mickael Grelat, Stephane Fuentes, Benjamin Blondel, et al.. Exposition des chirurgiens et des patients aux rayonnements ionisants pendant les procédures d'ostéosynthèse rachidienne postérieure percutanée : étude prospective de 100 patients. Revue de Chirurgie Orthopédique et Traumatologique, 2018, 104 (5), pp.433-439. 10.1016/j.rcot.2018.06.024 . hal-02137564

\author{
HAL Id: hal-02137564 \\ https://hal.science/hal-02137564
}

Submitted on 23 May 2019

HAL is a multi-disciplinary open access archive for the deposit and dissemination of scientific research documents, whether they are published or not. The documents may come from teaching and research institutions in France or abroad, or from public or private research centers.
L'archive ouverte pluridisciplinaire $\mathbf{H A L}$, est destinée au dépôt et à la diffusion de documents scientifiques de niveau recherche, publiés ou non, émanant des établissements d'enseignement et de recherche français ou étrangers, des laboratoires publics ou privés. 


\section{Exposition des chirurgiens et des patients aux rayonnements ionisants pendant les procédures d'ostéosynthèse rachidienne postérieure percutanée : étude prospective de 100 patients $^{\text {is }}$}

\section{Surgeon's and patient's radiation exposure during percutaneous thoracolumbar pedicle screw fixation: A prospective multicenter study of 100 cases}

Pascal Kouyoumdjïan ${ }^{\mathrm{a}}$, Guillaume Gras-Combe ${ }^{\mathrm{b}}$, Mickael Grelatc ${ }^{\mathrm{c}}$, Stéphane Fuentes ${ }^{\mathrm{d}}$, Benjamin Blondel ${ }^{\mathrm{e}}$, Patrick Tropiano ${ }^{\mathrm{e}}$, Fahed Zairi ${ }^{\mathrm{f}}$, Jacques Beaurain ${ }^{\mathrm{c}}$, Yann-Philippe Charles ${ }^{g}$, Alexandre Dhenin ${ }^{\mathrm{a}}$, Hassan Elfertit ${ }^{\mathrm{h}}$, Julien Le Roy ${ }^{\mathrm{i}}$, Joel Greffier ${ }^{\mathrm{j}}$, Nicolas Lonjon ${ }^{\mathrm{b}, *}$

a Orthopedic surgery, spine unit, university Montpellier 1, CHU Nîmes, hôpital Carémeau, 30029 Nîmes, France

${ }^{\mathrm{b}}$ Neurosurgery unit, CHU Montpellier, hôpital Gui-de-Chauliac, 34000 Montpellier, France

${ }^{\mathrm{c}}$ Neurosurgery unit, CHU Dijon-Bourgogne, 21000 Dijon, France

d Neurosurgery unit, hôpital la Timone, AP-HM, 13385 Marseille, France

e Orthopedic surgery, spine unit, hôpital la Timone, AP-HM, 13385 Marseille, France

${ }^{\mathrm{f}}$ Neurosurgery units, CHR, hôpital Roger-Salengro, 59037 Lille, France

g Orthopedic surgery, spine unit, CHRU Strasbourg, BP 426, 67091 Strasbourg, France

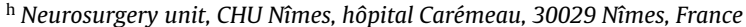

${ }^{i}$ Department of radiation physics, CHRU Montpellier, 34295 Montpellier, France

j Department of medical imaging, CHU Nîmes, hôpital Caremeau, 30029 Nîmes, France

\section{A B S T R A C T}

Hypothèse. - Les fixations percutanées par vis pédiculaire (FPVP) (FPVP) sont de plus en plus pratiquées en chirurgie du rachis, minimisant la morbidité via un moindre délabrement musculaire mais au prix d'un guidage fluoroscopique peropératoire générant une forte exposition aux rayonnements. Peu d'études ont été conduites pour les mesurer précisément. L'objectif de notre étude est de quantifier, lors d'une PPSF réa-lisé dans différents centres expérimentés respectant les recommandations actuelles de radioprotection, cette irradiation reçue au niveau du chirurgien et du patient.

Matériel et méthodes. - Nous avons inclus prospectivement 100 procédures de FPVP pour lesquelles nous avons relevé les doses d'irradiation de l'opérateur principal. Pour chaque intervention, les doses du rayonnement absorbées sur le corps entier, les cristallins et les extrémités ont été mesurées.

Résultats. - Nos résultats montrent une dose d'exposition moyenne par procédure sur le corps entier, les extrémités et les cristallins qui atteint respectivement, $1,7 \pm 2,8 \mu \mathrm{Sv}, 204,7 \pm 260,9 \mu \mathrm{Sv}$ et $30,5 \pm 25,9 \mu \mathrm{Sv}$.

Discussion. - Selon ses valeurs, l'exposition des extrémités et des cristallins du chirurgien dépassera la limite annuelle admise par la Commission internationale de protection radiologique (CIPR) après respectivement 2440 et 4840 procédures.

Conclusion. - Les directives récentes européennes vont réduire la dose d'exposition oculaire annuelle maximale de 150 à $20 \mathrm{mSv}$. Le nombre d'interventions chirurgicales pour ne pas atteindre le seuil oculaire, selon nos résultats, ne devra pas dépasser 645 procédures par an. Dans l'attente de la démocratisation des systèmes de neuronavigations, l'utilisation de la fluoroscopie conventionnelle expose les yeux en premier lieu et doivent donc être protégé par des verres plombés.

Niveau de preuve. - IV série de cas.

DOI de l'article original : https://doi.org/10.1016/j.otsr.2018.05.009.

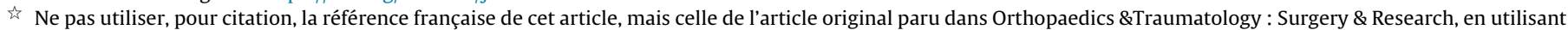
le DOI ci-dessus.

* Corresponding author Neurosurgery Unit, hôpital Gui-de-Chauliac, 80, avenue Augustin-Fliche, 34295 Montpellier cedex 5, France.

Adresse e-mail : nlonjon@gmail.com (N. Lonjon). 


\section{Introduction}

Les procédures mini-invasives utilisant une fixation percutanée par vis pédiculaire (FPVP) ont gagnées en popularité [1-5]. La nécessité d'un guidage fluoroscopique entraîne une exposition aux rayonnements importante à la fois pour le patient et l'équipe chirurgicale. L'exposition excessive à ces rayonnements $\mathrm{X}$ durant la réalisation de ces techniques, s'est avéré une préoccupation majeure de santé publique intéressant à la fois le chirurgien et le patient $[6,7]$. Elle dépend de divers facteurs : l'indice de masse corporelle (IMC) du patient, le niveau d'instrumentation, l'expérience du chirurgien et les caractéristiques techniques et réglages de l'arceau de bloc (C-arm) pendant la chirurgie. Des études récentes ont décrit des méthodes pour réduire cette exposition [7-14] tels l'utilisation de divers systèmes et supports d'imagerie (tomodensitométrie peropératoire (CT) combinée à la navigation) [15-17]. Ces systèmes sont coûteux et ne sont disponibles que dans une minorité de centres, laissant la majorité utiliser un ou deux C-arm conventionnels. Àce jour peu de données analysant l'exposition aux rayonnements, à la fois pour le patient et le chirurgien, pendant une procédure par FPVP, sont disponibles [6,10,14,18,19]. Cette étude clinique prospective multicentrique vise donc à quantifier l'exposition au rayonnement du chirurgien et du patient lors de l'utilisation en routine du FPVP dans des centres français référents en chirurgie rachidienne.

\section{Matériels et méthodes}

\subsection{Design de l'étude}

Cent patients consécutifs ont été inclus prospectivement dans cette étude de novembre 2014 à avril 2015 dans sept centres différents. Les patients ont été traités par FPVP sous contrôle fluoroscopique dans diverses indications. Dans chaque centre, l'expérience du chirurgien en FPVP dépassait 5 ans de pratique (plus de 30 FPVP/an). soit 14 chirurgiens (2 par centre) au total. Un seul C-arm a été utilisé dans 69 procédures, et 2 C-arm dans 31 cas. Différents dispositifs percutanés postérieurs ont été utilisés (Longitude Medtronic $^{\circledR}$, Mantis Stryker ${ }^{\circledR}$, Pathfinder Zimmer ${ }^{\circledR}$, Viper 2 Depuy ${ }^{\circledR}$ ) avec une technique de pose similaire. Tous les chirurgiens portaient un tablier plombé et une protection thyroïdienne plombée pendant les procédures, mais n'utilisaient pas de verres au plomb spécifiques ou d'équipements de protection collective.

\subsection{Exposition aux rayonnements du chirurgien}

L'exposition aux rayonnements du chirurgien a été recueillie comme déjà décrit [20,21]. Deux types de dosimètres ont été utilisés (Fig. 1) :

- des dosimètres thermoluminescents (TLD) passifs pour évaluer les doses équivalentes (Hp) du cristallin (Dosiris Cristallin, IRSN, Croisy sur Seine, France) et des extrémités (Bagues, IRSN, Croisy sur Seine, France), ce dernier étant positionné sur la face palmaire de l'annulaire de la main dominante. Les doses équivalentes aux extrémités ont été recueillies à une profondeur équivalente de tissu de $0,07 \mathrm{~mm}[\mathrm{Hp}(0,07)]$ et une profondeur de tissu de $3 \mathrm{~mm}$ [Hp (3)] pour le cristallin ;

- un dosimètre personnel électronique (EPD), dosimètre à lecture directe, porté sur la poitrine, sous le tablier de plomb. Ce dosimètre affichait le $\mathrm{Hp}$ à une profondeur équivalente de $10 \mathrm{~mm}[\mathrm{Hp}$ (10)] et cette valeur était considérée comme une estimation prudente de la dose efficace, indiquant une irradiation de l'ensemble du corps du chirurgien.

\subsection{L'analyse des données}

Les données ont été récupérées après chaque procédure en répertoriant le centre (localisation), le patient (âge, IMC), la chirurgie (indication, niveaux rachidiens, temps opératoire, arceau(s) utilisé(s), nombre de niveaux instrumentés, nombre de vis utilisées) et les indicateurs dosimétriques patient. Le produit de dose-surface (PDS, en cGy.cm²) et le temps fluoroscopique (TF, secondes) ont été collecté sur le C-arm et enregistrés à la fin de l'acte. L'irradiation du corps entier du chirurgien a été enregistrée à partir de l'EPD à la fin de chaque procédure. Pour chaque centre, les doses d'exposition au TLD ont été cumulées pendant la période d'inclusion. À la fin de l'étude, les TLD ont été renvoyées à l'IRSN (Croissy sur Seine, France) qui a effectué la mesure de la dose cumulée dans son laboratoire.

\subsection{Analyse statistique}

Les analyses statistiques ont été réalisées à l'aide des logiciels Matlab (MathWorks, Natick, MA USA) et BiostaTGV. Un test de corrélation de Pearson a été utilisé pour évaluer si le temps opératoire était corrélé au PDS, au TF et à Hp (10). Des comparaisons des doses mesurées [PDS, TF, Hp (10)] entre chaque procédure de
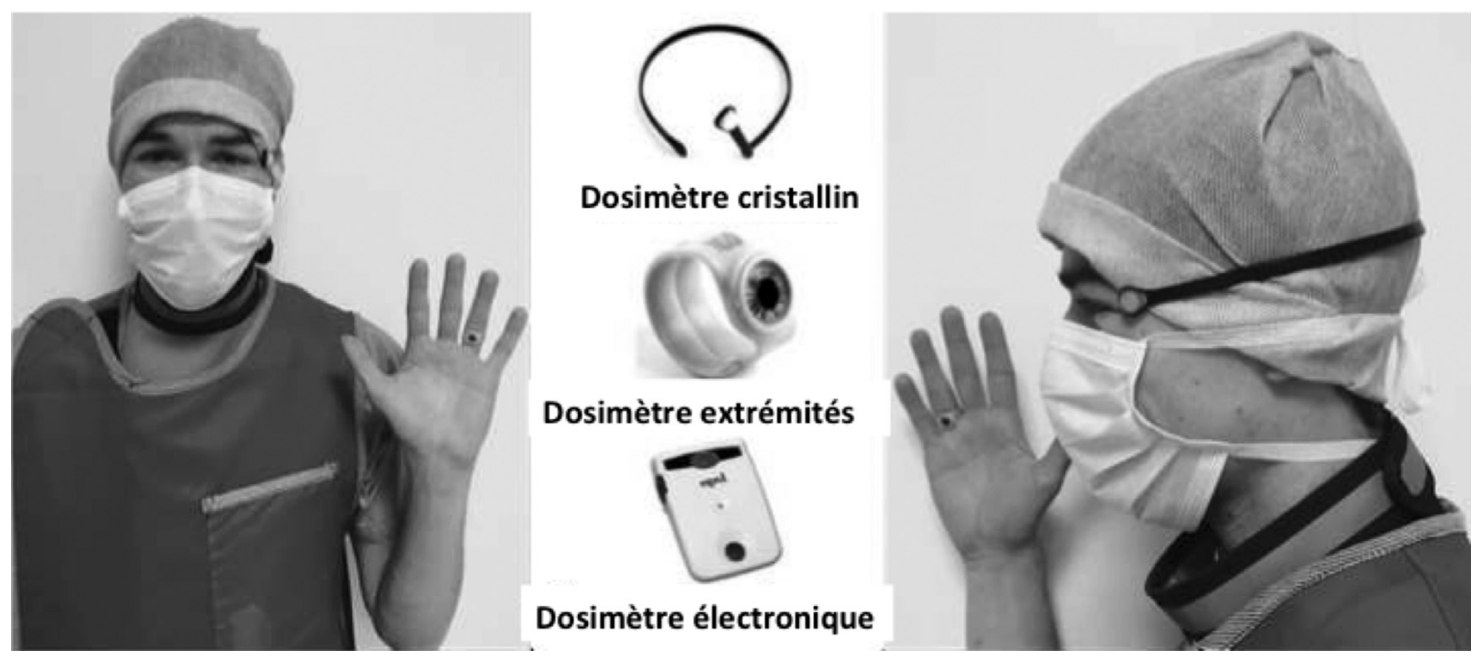

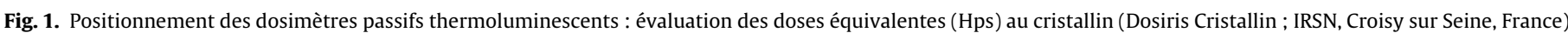
et extrémités (Bagues ; IRSN, Croisy sur Seine, France) des chirurgiens. 
fixation ont été effectuées en utilisant un test de Mann-Whitney Wilcoxon apparié. Les comparaisons avec des valeurs de $p<0,05$ ont été considérées comme des différences significatives. Afin de vérifier la distribution de l'échantillon pour chaque niveau et dans chaque centre, nous avons effectué le test ANOVA.

\section{Résultats}

\subsection{Population des patients}

Cent patients (Tableau 1) ont bénéficié d'une FPVP en tant que seule procédure de traitement. L'étiologie était traumatique dans 78 cas, dégénérative ou spondylolisthesis de bas grade (à distance temps antérieur) dans 17 cas métastatique dans 3 cas et une spondydiscite infectieuse dans 2 cas. Le nombre de vis pédiculaires implantées par procédure était de : 4 (67 patients), 8 (22 patients), 6 (7 patients), 7 ( 2 patients), 5 (1 patient), 10 (1 patient) et la longueur de la fixation postérieure était de 2, 3, 4, 5, 6, 7 niveaux respectivement dans $20,43,10,20,4$ et 3 cas.

\subsection{Exposition du patient}

La durée opératoire moyenne était de $72 \pm 35$ minutes et l'IMC moyen était de $25,2 \pm 4,7 \mathrm{~kg} / \mathrm{m}^{2}$, sans différence statistique entre les différents centres (Tableau 2). Dans les différents centres, le PDS et le TF médian ( $1^{\mathrm{e}}$ quartile ; $3^{\mathrm{e}}$ quartile) étaient respectivement de $763(418 ; 1315)$ cGy.cm² ${ }^{2}$ et $102(63 ; 187)$ secondes. Les données sont détaillées dans le Tableau 3. La médiane $\left(1^{\mathrm{e}}\right.$ quartile $; 3^{\mathrm{e}}$ quartile) de TF et du PDS par vis étaient respectivement de 19,2 (14,3 ; $38,8)$ s et de $142(74 ; 286)$ cGy.cm².

L'irradiation du patient était significativement plus élevée $(p<0,05)$ au niveau lombo-sacré qu'au niveau thoracique (760 $(437 ; 1200)$ et $1545(825 ; 2075)$ cGy.cm² $)$.

Les corrélations entre le temps opératoire et le PDS $(r=0,5454$, $p=5,32 \times 10-16)$, et le PDS et le TF $(r=0,5006 ; p=1,32 \times 10-17)$ étaient modérés. Celle entre le temps opératoire et le temps radioscopique $(r=0,2537 ; p=0,011)$ était faible. Les valeurs des PDS considérant le nombre d'arceau par procédure sont relatées dans le Tableau 4. Aucune différence statistique n'a été trouvée en fonction du nombre d'arceaux utilisés pendant la procédure. Les DAP par vis et par niveau anatomique en fonction du centre de la procédure sont rapportés dans le Tableau 5 . La valeur de la corrélation entre l'IMC et le PDS is faible $\left(\mathrm{R}^{2}<0,17\right)$ globalement et quel que soit le niveau (Fig. 2).

\subsection{Exposition du chirurgien}

La dose moyenne de rayonnement corporel du chirurgien par intervention était de $1,7 \pm 2,8 \mu \mathrm{Sv}$ et la médiane (1er quartile ; $3^{\mathrm{e}}$ quartile) était de $0,490(0,163 ; 1,505) \mu \mathrm{Sv}$. $\mathrm{HP}(0,07)$ moyenne

Tableau 1

Caractéristiques des patients.

\begin{tabular}{|c|c|c|c|c|c|}
\hline & Tous & Thoracique & Thoraco-Lombaire & Lombaire & Lombo-sacré \\
\hline Nombre & 100 & 9 & 45 & 31 & 15 \\
\hline $\operatorname{IMC}\left(\mathrm{kg} \cdot \mathrm{m}^{2}\right)$ & $25.2 \pm 4.7$ & $25.8 \pm$ & $24.2 \pm 5.0$ & $26.0 \pm 4.9$ & $25.7 \pm 3.2$ \\
\hline Âge & $55.1(19-96)$ & $69.0(47-89)$ & $53.7(19-96)$ & $53.9(19-87)$ & $51.9(31-82)$ \\
\hline \multirow[t]{2}{*}{ Sexe (f/m) } & $48 / 52$ & $3 / 6$ & $21 / 24$ & $17 / 14$ & $7 / 8$ \\
\hline & & Thoracique & Thoraco-lombaire & Lombaire & Lombo-sacré \\
\hline Centre 1 & & - & 11 & 7 & - \\
\hline Centre 2 & & - & 11 & 3 & 4 \\
\hline Centre 3 & & - & 2 & 9 & 5 \\
\hline Centre 4 & & 4 & - & 6 & 4 \\
\hline Centre 5 & & 3 & 7 & 3 & 2 \\
\hline Centre 6 & & 2 & 9 & 2 & - \\
\hline Centre 7 & & - & 5 & 1 & - \\
\hline
\end{tabular}

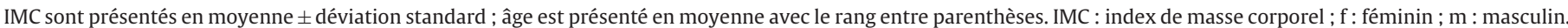

Tableau 2

Données patients, temps opératoire et indicateur dosimétrique par centre.

\begin{tabular}{|c|c|c|c|c|}
\hline & $\operatorname{IMC}\left(\mathrm{kg} / \mathrm{m}^{2}\right)$ & Durée opératoire (min) & PDS (cGy.cm²) & $\mathrm{TF}$ (secondes) \\
\hline Centre 1 & $25,5 \pm 5,0$ & $107 \pm 30$ & $1604(1260 ; 1860)$ & $233(193 ; 311)$ \\
\hline Centre 2 & $24,6 \pm 3,5$ & $59 \pm 25$ & $277(227 ; 693)$ & $60(57 ; 87)$ \\
\hline Centre 3 & $25,4 \pm 2,9$ & $48 \pm 16$ & $834(512 ; 2230)$ & $71(39 ; 130)$ \\
\hline Centre 4 & $26,5 \pm 5,4$ & $58 \pm 26$ & $842(564 ; 1140)$ & $83(73 ; 115)$ \\
\hline Centre 5 & $23,5 \pm 3,7$ & $90 \pm 37$ & $1150(542 ; 1732)$ & $194(140 ; 289)$ \\
\hline Centre 6 & $26,0 \pm 7,8$ & $56 \pm 27$ & $425(261 ; 467)$ & $108(77 ; 145)$ \\
\hline Centre 7 & $24,2 \pm 2,3$ & $108 \pm 26$ & $625(420 ; 732)$ & $78(69 ; 92)$ \\
\hline Tous les centres & $25,2 \pm 4,7$ & $72 \pm 35$ & $763(418 ; 1315)$ & $102(63 ; 187)$ \\
\hline
\end{tabular}

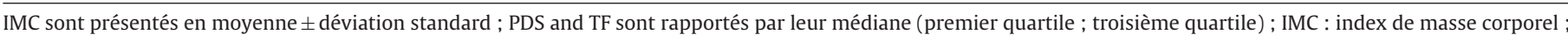
PDS : produit dose surface ; TF : temps de fluoroscopie.

Tableau 3

Données du patient, temps opératoire et indicateur dosimétrique par niveau anatomique de la procédure.

\begin{tabular}{|c|c|c|c|c|}
\hline & IMC $\left(\mathrm{kg} / \mathrm{m}^{2}\right)$ & Durée opératoire (min) & PDS $\left(\mathrm{cGy} \cdot \mathrm{cm}^{2}\right)$ & TF (secondes) \\
\hline Thoracique & $25,8 \pm 4,5$ & $84 \pm 29$ & $760(437 ; 1200)$ & $138(90 ; 147)$ \\
\hline Thoraco-Lombaire & $24,3 \pm 4,9$ & $77 \pm 39$ & $627(256 ; 1060)$ & $120(65 ; 199)$ \\
\hline Lombaire & $26,0 \pm 4,9$ & $63 \pm 32$ & $834(464 ; 1265)$ & $80(58 ; 173)$ \\
\hline Lombo-sacré & $25,7 \pm 3,2$ & $70 \pm 30$ & $1545(825 ; 2075)$ & $109(67 ; 197)$ \\
\hline Tous les centres & $25,2 \pm 4,7$ & $72 \pm 35$ & $763(418 ; 1315)$ & $102(63 ; 187)$ \\
\hline
\end{tabular}

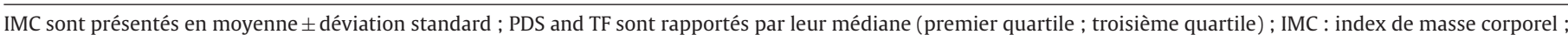
PDS : produit dose surface ; TF : temps de fluoroscopie. 
Tableau 4

PDS par nombre de C-arm utilisé par procédure.

\begin{tabular}{|c|c|c|c|c|c|c|}
\hline & \multicolumn{2}{|c|}{ Nombre de patient } & \multicolumn{2}{|c|}{ PDS (mGy.cm²) } & \multicolumn{2}{|c|}{ Durée opératoire (min) } \\
\hline & $1 \mathrm{C}$-arm & 2 C-arm & $1 \mathrm{C}$-arm & 2 C-arm & $1 \mathrm{C}$-arm & $2 \mathrm{C}$-arm \\
\hline Thoracique & 7 & 2 & $1089 \pm 705$ & $676 \pm 454$ & $79 \pm 21$ & $96 \pm 48$ \\
\hline Thoraco-lombaire & 27 & 18 & $417 \pm 267$ & $1435 \pm 908$ & $60 \pm 33$ & $103 \pm 32$ \\
\hline Lombaire & 23 & 8 & $854 \pm 856$ & $1619 \pm 848$ & $50 \pm 19$ & $96 \pm 37$ \\
\hline Lombo-sacré & 12 & 3 & $2118 \pm 2745$ & $1732 \pm 264$ & $64 \pm 27$ & $105 \pm 21$ \\
\hline Total & 69 & 31 & $934 \pm 1409$ & $1434 \pm 851$ & $59 \pm 28$ & $101 \pm 33$ \\
\hline
\end{tabular}

Résultats exprimés par la moyenne \pm la déviation standard.

Tableau 5

PDS (mGy. $\mathrm{cm}^{2}$ ) par vis considérant le niveau anatomique de la chirurgie et le centre de prise en charge.

\begin{tabular}{|c|c|c|c|c|c|}
\hline & Thoracique & Thoraco-lombaire & Lombaire & Lombo-sacré & Total \\
\hline Centre 1 & - & $270 \pm 87$ & $451 \pm 198$ & - & $335 \pm 157$ \\
\hline Centre 2 & 95 & $55 \pm 26$ & $91 \pm 28$ & $321 \pm 131$ & $108 \pm 111$ \\
\hline Centre 3 & - & $141 \pm 95$ & $172 \pm 73$ & $655 \pm 89$ & $271 \pm 221$ \\
\hline Centre 4 & $92 \pm 69$ & 210,5 & $195 \pm 84$ & $183 \pm 157$ & $166 \pm 109$ \\
\hline Centre 5 & $300 \pm 264$ & $282 \pm 355$ & $218 \pm 228$ & 479,8 & $292 \pm 281$ \\
\hline Centre 6 & $48 \pm 7$ & $90 \pm 55$ & $54 \pm 33$ & - & $74 \pm 47$ \\
\hline Centre 7 & - & $103 \pm 63$ & 69,7 & - & $97 \pm 58$ \\
\hline Total & $146 \pm 169$ & $157 \pm 169$ & $209 \pm 161$ & $360 \pm 231$ & $199 \pm 186$ \\
\hline
\end{tabular}

Résultats exprimés par la moyenne \pm la déviation standard.

Tableau 6

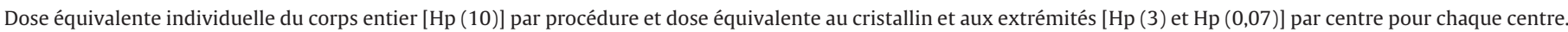

\begin{tabular}{|c|c|c|c|}
\hline & \multirow{2}{*}{$\begin{array}{l}\text { Dosimètres opérationnels (EPD) } \\
\text { Corps entier }\end{array}$} & \multicolumn{2}{|c|}{ Dosimètres personnels (TLD) } \\
\hline & & Cristallin & Extrémités \\
\hline & $\mathrm{Hp}(10)(\mu \mathrm{Sv})$ & $\mathrm{Hp}(3)(\mu \mathrm{Sv})$ & $\mathrm{Hp}(0,07)(\mu \mathrm{Sv})$ \\
\hline Centre 1 & $0,310(0,170 ; 0,530)$ & 75,6 & 81,1 \\
\hline Centre 2 & $0,660(0,380 ; 1,190)$ & 14,4 & 85,6 \\
\hline Centre 3 & $0,001(0,001 ; 0,002)$ & 53,5 & 324,1 \\
\hline Centre 4 & $0,845(0,245 ; 3,855)$ & 25,6 & 124,4 \\
\hline Centre 5 & $1,000(0,310 ; 7,300)$ & 32,0 & 751,3 \\
\hline Centre 6 & $0,001(0,001 ; 0,002)$ & 7,7 & 28,1 \\
\hline Centre 7 & $0,530(0,298 ; 1,385)$ & 5,5 & 50,0 \\
\hline Tous les Centres & $0,490(0,163 ; 1,505)$ & $30,5 \pm 25,9$ & $204,7 \pm 260,9$ \\
\hline
\end{tabular}

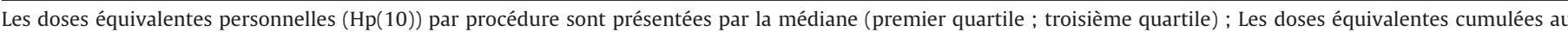

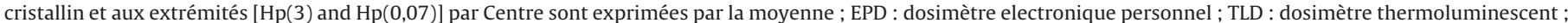
$\mathrm{Hp}$ : dose equivalente personnel.

Tableau 7

Dose équivalente individuelle du corps entier [Hp (10)] par procédure pour chaque niveau de la chirurgie.

\begin{tabular}{ll}
\hline & Dosimètre opérationnel (EPD) \\
\hline Thoracique & Corps entier $\operatorname{Hp}(10)(\mu \mathrm{Sv})$ \\
Thoraco-Lombaire & $0,805(0,103,1,063)$ \\
Lombaire & $0,380(0,165,0,690)$ \\
Lombo-sacré & $0,530(0,140,3,090)$ \\
Tous les centres & $1,920(0,690,3,113)$ \\
\hline
\end{tabular}

Les résultats sont rapportés par la médiane (premier quartile ; troisième quartile) ; $\mathrm{Hp}$ : dose équivalente personnelle.

pour le cristallin était de $30,5 \pm 25,9 \mu \mathrm{Sv}$ et de $204,7 \pm 260,9 \mu \mathrm{Sv}$ pour les extrémités. $\mathrm{Hp}(3)$ moyenne de chaque centre variait entre 0,6 et $16,8 \mu \mathrm{Sv}$ et le $\mathrm{Hp}(0,07)$ moyenne entre 28,1 et $751,3 \mu \mathrm{Sv}$ (Tableau 6).

La dose efficace moyenne reçue par les chirurgiens par procédure variait avec le niveau anatomique, tendant à augmenter en lombaire et lombo-sacrée $(1,920(0,690 ; 3,113) \mu S v$ pour lombosacré contre $0,803(0,103 ; 1,063) \mu S v$ pour thoracique (Tableau 7$)$. La corrélation entre le temps opératoire et $\operatorname{Hp}(10)(r=0,1583$; $p=0,0181$ ) était faible.

\section{Discussion}

Les procédures percutanées certes fiables[22] exposent les patients et les chirurgiens à une irradiation peropératoire plus élevée qu'à ciel ouvert.

Très peu travaux référencés ont étudié l'exposition aux rayonnements pendant les FPVP[6]. Les effets indésirables des rayonnements ionisants [18] déterministes ou stochastiques. et leur sous-estimation peuvent entraîner des risques de cancer cinq fois plus élevés pour les chirurgiens exposés [1,23]. Les chirurgiens du rachis sont exposés à un niveau de rayonnement 10 fois plus élevé que les chirurgiens orthopédiques [24] notamment au cours des procédures percutanées mini-invasives [6,14]. Bronsard et col. ont comparé la fixation ouverte classique lombaire avec la fixation fermée [6], montrant que le PDS et le temps de fluoroscopie étaient respectivement de $267,7(58,3-2054,0) \mathrm{cGy}_{\mathrm{cm}} \mathrm{cm}^{2}$ et de $29,5(13,2-83,1)$ secondes en chirurgie ouverte contre 868,2 $(191,6-2885,2)$ cGy.cm ${ }^{2}$ et $139,7(36,0-388,1)$ secondes en procédure percutanée. Cependant les données publiées restent pauvres.

À l'exception de la région lombo-sacrée, le temps de fluoroscopie et le PDS, et donc les valeurs de la dose efficace pour les patients, étaient inférieures à celles des études antérieures[25-27] ou du moins similaires considérant la moyenne du TF et du PDS pour chaque vis[6,14,18]. L'épaisseur du volume de diffusion est plus 


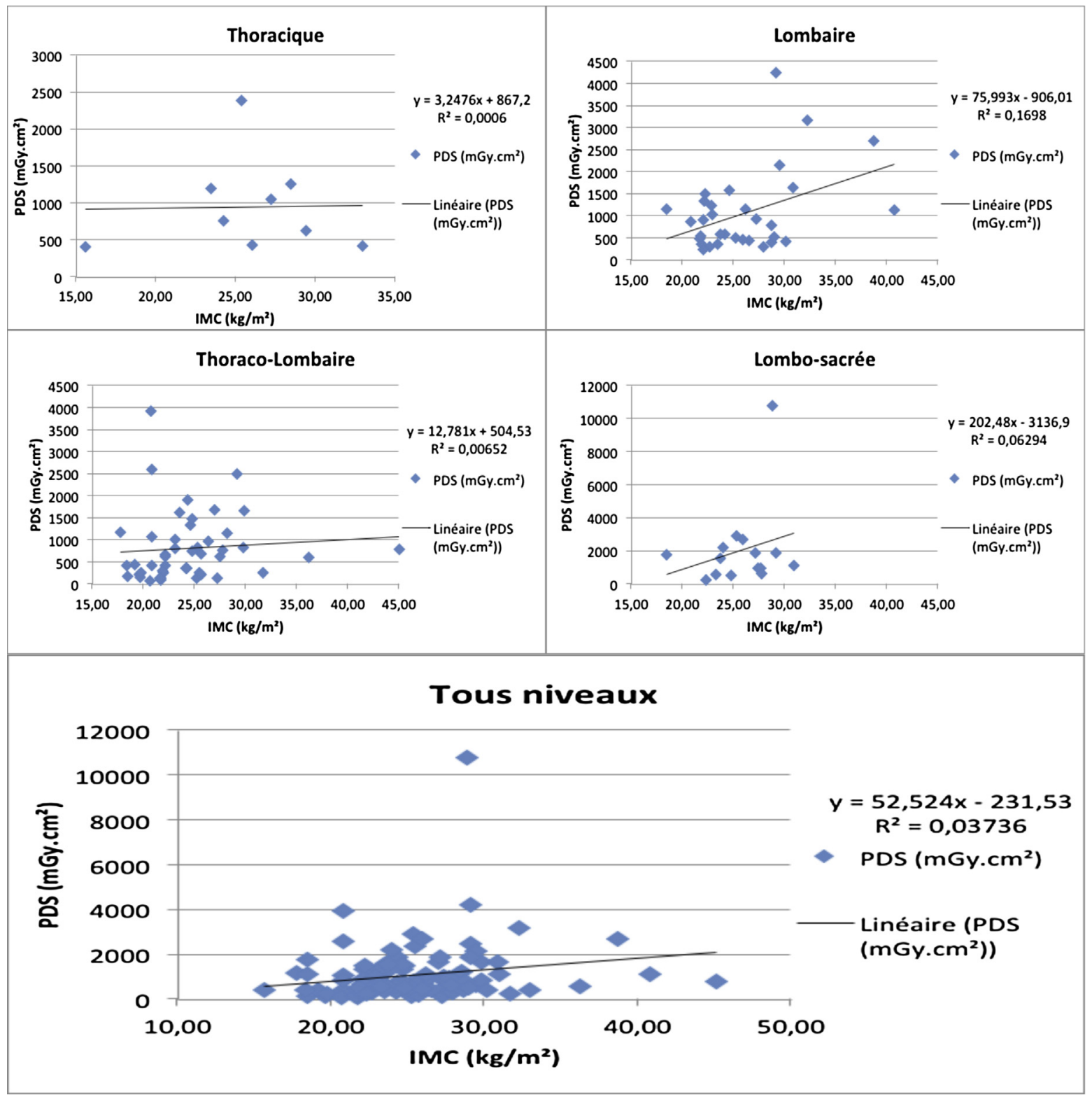

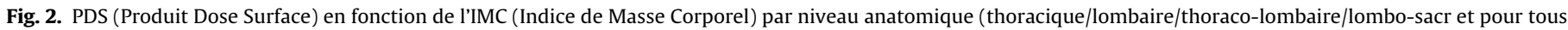
les niveaux.

élevée au niveau lombaire et lombo-sacré qu'au niveau thoracique, entraînant une augmentation de la quantité de rayonnement $(\mathrm{kV}$ et $\mathrm{mA}$ ) à la sortie du tube et donc du débit de dose, ce qui augmente le PDS.

Seules les procédures réalisées par des chirurgiens expérimentés ont été inclues éliminant ainsi l'effet de la courbe d'apprentissage et homogénéisant notre étude. Nous n'avons trouvé aucun effet centre statistiquement significatif.

Cette photographie de l'exposition d'une grande hétérogénéité en fonction des centres et des niveaux opérés. Son origine est multifactorielle (type de scopie, rigueur du respect des règles de radioprotection, localisation de la chirurgie, chirurgien, difficulté de la chirurgie, type de protections utilisées etc.). Cependant ce travail a pour vertu de les globaliser et de déterminer un ordre de grandeur de l'irradiation subie à la fois par le chirurgien et le patient.

Plusieurs mesures permettant de réduire le temps et la dose de rayonnement.[28-36].

La limite de dose annuelle d'exposition du chirurgien recommandée par le Guide de la Commission internationale de protection radiologique (CIPR) est de $500 \mathrm{mSv}$ pour les extrémités et de $150 \mathrm{mSv}$ pour les yeux. Dans notre étude, la dose moyenne de rayonnement au cristallin et aux extrémités par intervention était respectivement de $0,031 \pm 0,026 \mathrm{mSv}$ et de $0,205 \pm 0,261 \mathrm{mSv}$ et inférieure à l'étude précédente sur la procédure de vertébroplastie percutanée [37] et plus faible comparée aux procédures de cyphoplastie [30]. Selon nos résultats, la dose d'exposition aux extrémités et au cristallin dépasserait la limite annuelle pour un nombre de procédures respectivement de 4840 et 2440.

Jacob et al. [38] ont rapporté dans leur série que les opacités sous-capsulaires postérieures du cristallin étaient significativement plus fréquentes chez les cardiologues interventionnels (17\% vs $5 \%, p=0,006$ ), correspondant à un OR brut $=3,89$. En partie liée à ce risque, et pour rester en ligne avec les orientations de la CIPR, une récente directive européenne (2013/59/EURATOM) a abaissé la limite annuelle à $20 \mathrm{mSv}$. En utilisant ce seuil, la dose d'exposition au cristallin dépasserait la limite annuelle après 645 procédures.

En plus des précautions de sécurité individuelles habituelles, l'utilisation de la fluoroscopie pulsée, la collimation, la 
réduction manuelle du débit de dose, l'utilisation d'images enregistrées au lieu de radiographies supplémentaires et la distance source-détecteur optimisée sont également recommandées. De nombreuses études ont mis en évidence l'intérêt de la navigation fluoroscopique [16,40-44] ou de nouveaux dispositifs expérimentaux [9,39], notamment à l'étage sacré [12],. La fluoroscopie 2D conventionnelle reste actuellement la méthode de guidage d'image la plus utilisée dans tous nos centres en tout cas pendant la période de cette étude.

Notre étude avait plusieurs limites. L'analyse statistique manquait de puissance en raison du faible nombre de patients par procédure et centre, et des influences croisées entre le centre et le niveau de la chirurgie. En outre, les procédures étaient hétérogènes en termes de dispositifs percutanés et de système de fluoroscopie, mais aussi probablement en termes d'utilisation de la scopie par le chirurgien même si expérimenté. Cette étude n'a pas intégré les chirurgiens juniors potentiellement les plus pourvoyeurs d'irradiation.

\section{Conclusion}

Nous rapportons les résultats de l'exposition du chirurgien et du patient dans 7 centres. Les récentes recommandations de la CIPR et la directive européenne limitent la dose annuelle à l'œil à moins de $20 \mathrm{mSv}$, ce qui limite le nombre de procédures chirurgicales selon nos données à 645 procédures par an. Alors que les mesures de radioprotection actuelles semblent adéquates sur la base de la faible dose enregistrée pour les chirurgiens et les patients, celles-ci doivent être strictement observées et suivies à l'avenir.

\section{Source de financement}

Nous avons reçu pour cette recherché des fonds de la Société française de chirurgie rachidienne. Ces fonds ont été utilisés pour acheter les matériels d'équipement (dosimètre thermoluminescent).

\section{Contribution des auteurs}

N.L. et P.K. ont conçu le modèle, le cadre computationnel et écrit l'article.

N.L et P.K. ont dirigé le projet et écrit l'article.

J.G et J.L.R ont analysé les data, contribué à la conception de la recherche, à l'analyse des résultats et à l'écriture du manuscrit.

G.G.C, M.G, S.F, B.B, P.T, F.Z, J.B, Y.P. C, A.D, H.E ont traité les données expérimentales, aidé à interpréter les résultats, effectué la mise en œuvre.

\section{Remerciements}

Nous souhaitons remercier Sarah Kabani pour son aide précieuse.

\section{Déclaration de liens d'intérêts}

Les auteurs déclarent ne pas avoir de liens d'intérêts.

\section{Références}

[1] Wiesner L, Kothe R, Schulitz KP, et al. Clinical evaluation and computed tomography scan analysis of screw tracts after percutaneous insertion of pedicle screws in the lumbar spine. Spine 2000;25:615-21.

[2] Giorgi H, Prebet R, Delhaye M, et al. Minimally invasive posterior transforaminal lumbar interbody fusion: One-year postoperative morbidity, clinical and radiological results of a prospective multicenter study of 182 cases. OTSR 2015;101:S241-5.
[3] Grass R, Biewener A, Dickopf A, et al. Percutaneous dorsal versus open instrumentation for fractures of the thoracolumbar border. A comparative, prospective study. Unfallchirurg 2006;109:297-305.

[4] Fuentes S, Blondel B, Metellus P, et al. Percutaneous kyphoplasty and pedicle screw fixation for the management of thoraco-lumbar burst fractures. Eur Spine J 2010;19:1281-7.

[5] McAnany SJ, Overley SC, Kim JS, et al. Open versus minimally invasive fixation techniques for thoracolumbar trauma: a meta-analysis. Global Spine J 2016;6:186-94.

[6] Bronsard N, Boli T, Challali M, et al. Comparison between percutaneous and traditional 19 fixation of lumbar spine fracture: intraoperative radiation exposure levels and outcomes. OTSR 2013;99:162-8.

[7] Funao H, Ishii K, Momoshima S, et al. Surgeons' exposure to radiation in singleand multi-level minimally invasive transforaminal lumbar interbody fusion ; a prospective study. PloS one 2014;9:e95233.

[8] Muller MC, Welle K, Strauss A, et al. Real-time dosimetry reduces radiation exposure of 25 orthopaedic surgeons. OTSR 2014;100:947-51.

[9] Fraser JF, Von Jako R, Carrino JA, et al. Electromagnetic navigation in minimally invasive spine surgery: results of a cadaveric study to evaluate percutaneous pedicle screw insertion. SAS journal 2008;2:43-7.

[10] Gu G, Zhang H, He S, et al. Percutaneous pedicle screw placement in the lumbar spine: a comparison study between the novel guidance system and the conventional fluoroscopy method. J Spinal Disord Tech 2015;28: E522-7.

[11] Houten JK, Nasser R, Baxi N. Clinical assessment of percutaneous lumbar pedicle screw placement using theO-arm multidimensional surgical imaging system. Neurosurgery 2012;70:990-5.

[12] Kraus MD, Krischak G, Keppler P, et al. Can computer-assisted surgery reduce the effective dose for spinal fusion and sacroiliac screw insertion? Clin Orthop Relat Res 2010;468:2419-29.

[13] Tonetti J, Carrat L, Blendea S, et al. Clinical validation of computer assisted pelvic surgery using ultrasound. A percutaneous safe technique with low radiation exposure. Stud Health Technol Inform 2001;81:515-20.

[14] Srinivasan D, Than KD, Wang AC, et al. Radiation safety and spine surgery: systematic review of exposure limits and methods to minimize radiation exposure. World Neurosurg 2014;82:1337-43.

[15] Foley KT, Simon DA, Rampersaud YR. Virtual fluoroscopy: computer-assisted fluoroscopic navigation. Spine 2001;26:347-51.

[16] Yang BP, Wahl MM, Idler CS. Percutaneous lumbar pedicle screw placement aided by computer-assisted fluoroscopy-based navigation: perioperative results of a prospective, comparative, multicenter study. Spine 2012;37:2055-60.

[17] Luciano CJ, Banerjee PP, Sorenson JM, et al. Percutaneous spinal fixation simulation with virtual reality and haptics. Neurosurgery 2013;72:89-96.

[18] Mroz TE, Abdullah KG, Steinmetz MP, et al. Radiation exposure to the surgeon during percutaneous pedicle screw placement. J Spinal Disord Tech $2011 ; 24: 264-7$

[19] Slomczykowski M, Roberto M, Schneeberger P, et al. Radiation dose for pedicle screw insertion. Fluoroscopic method versus computer-assisted surgery. Spine 1999;24:975-82 [discussion 83].

[20] Grelat M, Greffier J, Sabatier P, et al. Assessment of the Radiation Exposure of Surgeons and Patients During a Lumbar Microdiskectomy and a Cervical Microdiskectomy: A French Prospective Multicenter Study. World Neurosurg 2016;89:329-36.

[21] Lonjon N, Kouyoumdjian P, Prieto M, et al. Early functional outcomes and histological analysis after spinal cord compression injury in rats. J Neurosurgery 2010;12:106-13.

[22] Baird EO, McAnany SJ, Overley S, et al. Accuracy of percutaneous pedicle screw placement: does training level matter? Clin Spine Surg 2017;30(6) E748-53.

[23] Mastrangelo G, Fedeli U, Fadda E, et al. Increased cancer risk among surgeons in an orthopaedic hospital. Occup Med 2005;55:498-500.

[24] Rampersaud YR, Foley KT, Shen AC, et al. Radiation exposure to the spine surgeon during fluoroscopically assisted pedicle screw insertion. Spine 2000;25:2637-45.

[25] Bindal RK, Glaze S, Ognoskie M, et al. Surgeon and patient radiation exposure in minimally invasive transforaminal lumbar interbody fusion. J Neurosurg 2008;9:570-3.

[26] Perisinakis K, Theocharopoulos N, Damilakis J, et al. Estimation of patient dose and associated radiogenic risks from fluoroscopically guided pedicle screw insertion. Spine 2004;29:1555-60.

[27] Solomiichuk V, Fleischhammer J, Molliqaj G, et al. Robotic versus fluoroscopyguided pedicle screw insertion for metastatic spinal disease: a matched-cohor comparison. Neurosurg Focus 2017;42:E13.

[28] Waddell BS, Waddell WH, Godoy G, et al. Comparison of ocular radiation exposure utilizing three types of leaded glasses. Spine 2016;41:E231-6.

[29] Villard J, Ryang YM, Demetriades AK, et al. Radiation exposure to the surgeon and the patient during posterior lumbar spinal instrumentation: a prospective randomized comparison of navigated versus non-navigated freehand techniques. Spine 2014;39:1004-9.

[30] Wang J, Liu H, Zhang K, et al. Reducing radiation exposure during kyphoplasty with the use of a remote control injection system: a prospective study. Spine 2015;40:E127-32.

[31] Wood MJ, Mannion RJ. Improving accuracy and reducing radiation exposure in minimally invasive lumbar interbody fusion. J Neurosurg 2010;12:533-9 [Spine]. 
[32] Wu H, Gao ZL, Lu ZW, et al. Radiation exposure to spine surgeon: a comparison of computer-assisted navigation and conventional technique. China J Orthop Traumatology 2009;22:874-6.

[33] Theocharopoulos N, Damilakis J, Perisinakis K, et al. Occupational gonadal and embryo/fetal doses from fluoroscopically assisted surgical treatments of spinal disorders. Spine 2004;29:2573-80.

[34] Goodman BS, Carnel CT, Mallempati S, et al. Reduction in average fluoroscopic exposure times for interventional spinal procedures through the use of pulsed and low-dose image settings. Am J Phys Med Rehabil 2011;90:908-12.

[35] Li YY, Huang TJ, Cheng CC, et al. Comparing radiation exposure during percutaneous vertebroplasty using one- vs. two-fluoroscopic technique. BMC Musculoskelet Disord 2013;14:38

[36] Singer G. Occupational radiation exposure to the surgeon. J American Acad Orthop Surg 2005;13:69-76.

[37] Harstall R, Heini PF, Mini RL, et al. Radiation exposure to the surgeon during fluoroscopically assisted percutaneous vertebroplasty: a prospective study. Spine 2005;30:1893-8.

[38] Jacob S, Boveda S, Bar O, et al. Interventional cardiologists and risk of radiationinduced cataract: results of a French multicenter observational study. Int J Cardiol 2013;167:1843-7.
[39] Zhang L, Zhou X, Cai X, et al. Reduction in radiation during percutaneous lumbar pedicle screw placement using a new device. Minim Invasive Ther Allied Technol 2014;23:173-8.

[40] Moses ZB, Mayer RR, Strickland BA, et al. Neuronavigation in minimally invasive spine surgery. Neurosurg Focus 2013;35:E12.

[41] Powell MF, DiNobile D, Reddy AS. C-arm fluoroscopic cone beam CT for guidance of minimally invasive spine interventions. Pain Physician 2010;13: $51-9$.

[42] Ruatti S, Dubois C, Chipon E, et al. Interest of intra-operative 3D imaging in spine surgery: a prospective randomized study. Eur Spine J 2016;25: 1738-44.

[43] Schouten R, Lee R, Boyd M, et al. Intra-operative cone-beam CT (O-arm) and stereotactic navigation in acute spinal trauma surgery. J Clin Neurosci 2012;19:1137-43.

[44] Stadler 3rd JA, Dahdaleh NS, Smith ZA, et al. Intraoperative navigation in minimally invasive transforaminal lumbar interbody fusion and lateral interbody fusion. Neurosurg Clin North Am 2014;25:377-82. 